\title{
Challenges in the management of pediatric asthma
}

\author{
Ai Huan Chen, MD \\ Guangzhou Institute of Respiratory Diseases, \\ First Affiliated Hospital, Guangzhou Medical College, China
}

Inhaled corticosteroid(ICS) remains the first line maintenance therapy for persistent asthma in both children and adults, but it can not meet all the needs in the management of paediatric asthma. Firstly, not all paediatric asthmatics show good response to ICS, there is a considerable variability of response to ICS among individuals. In some asthmatic children, asthma can not be well controlled even with high dose of ICS. Secondly, several key problems remain uncertain in the management of paediatric asthma with ICS, such as how to predict the individual response to ICS and how long ICS has to be used. In the Childhood Asthma Management Program (CAMP) study of school-aged children with mild to moderate asthma, treatment with ICS is associated with significant improvements in most features of asthma control, but does not affect the natural course of the disease. The improvements disappear within months after ICS is discontinued. For asthmatic children who are not adequately controlled with ICS alone, a higher dose of ICS is one choice of treatment to be considered, but adding another class of controller drug (such as Long-acting inhaled ß2-agonists, leukotriene modifiers, sustained-release theophylline) is preferable. However, which is the best choice as add-on therapy in combination with ICS in paediatric asthma remains unclear. Adding longacting inhaled $ß 2$-agonists (LABA) on ICS therapy is so far the most effective combination therapy in adult asthma, but there is insufficient evidence to support a general recommendation for their use in paediatric asthma. A few of studies have suggested that leukotriene modifiers may be more effective in children younger than 10 years. Moreover, the response to these various add-on medications differs from patient to patient. The choice should be individually tailored. Thirdly, wheezing is a common symptom in young children especially in infants, many children who wheeze during the first 2 to 3 years of life are transient wheezers, they do not wheeze after the age of 3 years. But some of them will be persistent wheezers and subsequently develop to chronic asthma. How to identify the wheezing infants and young children who will go on to develop asthma? And which measures could effectively modify this natural course of asthma in young children at high risk for subsequent development of asthma? Lastly, how to increase the lung deposition of ICS and how to improve the compliance of the asthmatic children on ICS also are big challenges in the management of paediatric asthma. 


\section{Curriculum Vitae}

Name: Chen Ai Huan ( 陈爱欢)

Employer: Guangzhou Institute of Respiratory Diseases

Titles: Professor, Postgraduate tutor, Consultant

Academic degree: $\mathrm{MD}$

\section{Professional experiences:}

Aug 2004-present Professor, Guangzhou Institute of Respiratory Diseases, The First Affiliated Hospital of Guangzhou Medical College

Dec 2003-Jul 2004 Professor, Pediatric department, The First Affiliated Hospital of Guangzhou Medical College

Dec 1998-Nov 2003 Associate professor, Pediatric department, The First Affiliated Hospital of Guangzhou Medical College

Jul 1987-Nov 1998 Resident, lecturer, The First Affiliated Hospital of Guangzhou Medical College

\section{Education:}

Sep 1996-Jun 1999 Postgraduate, Guangzhou Institute of Respiratory Diseases

Sep 1982-Aug 1987 Undergraduate, Medical Faculty, Guangzhou Medical College

\section{Research Interests:}

1. The mechanisms and pharmacology of allergic diseases especially asthma and rhinitis in children.

2. The mechanism and pharmacology of obstructive sleep apnea/hypopnea syndrome in children.

3. Lung function and respiratory mechanics in children. 